\title{
Logistics Modeling for Lunar Exploration Systems
}

\author{
Mark R. Andraschko ${ }^{1}$ and R. Gabe Merrill ${ }^{2}$ \\ Analytical Mechanics Associates, Inc, Hampton, VA, 23666 \\ and \\ Kevin D. Earle ${ }^{3}$ \\ Systems Analysis \& Concepts Directorate, NASA Langley Research Center, Hampton, VA, 23681
}

\begin{abstract}
The extensive logistics required to support extended crewed operations in space make effective modeling of logistics requirements and deployment critical to predicting the behavior of human lunar exploration systems. This paper discusses the software that has been developed as part of the Campaign Manifest Analysis Tool in support of strategic analysis activities under the Constellation Architecture Team - Lunar. The described logistics module enables definition of logistics requirements across multiple surface locations and allows for the transfer of logistics between those locations. A key feature of the module is the loading algorithm that is used to efficiently load logistics by type into carriers and then onto landers. Attention is given to the capabilities and limitations of this loading algorithm, particularly with regard to surface transfers. These capabilities are described within the context of the object-oriented software implementation, with details provided on the applicability of using this approach to model other human exploration scenarios. Some challenges of incorporating probabilistics into this type of logistics analysis model are discussed at a high level.
\end{abstract}

\section{Introduction}

$\mathrm{T}$ o support NASA's development of an exploration system capable of establishing an extended human presence on the Moon, the impacts of variations in system parameters must be studied at an architecture level to inform Constellation Program senior management on the benefit, viability, affordability, and robustness of system design choices. The Constellation Program's Strategic Analysis Team (SAT) performs this analysis both deterministically and probabilistically to combine performance, risk, cost, and value into a set of Figures of Merit that can be used to compare the overall benefit of a set of lunar exploration systems. As part of that effort, the modeling must account for the logistics to enable crew habitation, element maintenance, and science activities. The overall value of a human lunar return and subsequent extended duration surface stays is significantly driven by the logistics requirements, packaging design, and re-supply methodology As such, one of the primary goals of strategic analysis is to provide an integrated assessment of the logistics system over the exploration system life-cycle to support strategic decision making ${ }^{1}$.

The Campaign Manifest Analysis Tool (CMAT) is a deterministic software model that is used by the SAT to perform the initial studies of exploration options. CMAT allows for quick creation of scenarios and sensitivities, enabling rapid trade space exploration. The focus of CMAT is the delivery of elements and goods to locations on the lunar surface, with the phasing of those deliveries and the pace of the surface architecture build-up being driven largely by the amount of mass that the lunar transportation system is capable of delivering to those specific locations. It does not perform any element sizing or transportation system analysis. Rather, those parameters are provided as inputs by teams of subject matter experts using their models and analysis. Users define a series of missions by specifying parameters such as arrival dates, delivery mass, and manifested surface elements in the CMAT Core Module. The defined exploration scenario is then used by the CMAT Logistics Module to determine

\footnotetext{
${ }^{1}$ Project Engineer, 303 Butler Farm Rd, Suite 104A, AIAA Member.

2 Project Engineer, 303 Butler Farm Rd, Suite 104A, AIAA Member.

${ }^{3}$ Aerospace Engineer, Space Mission Analysis Branch, MS 462, 1 N. Dryden Street, not an AIAA Member.
} 
the logistics required for a given exploration scenario and then, to automatically load those logistics onto the defined missions. The majority of this paper will focus on the calculations performed by the Logistics Module to determine the logistics requirements and the logistics loading algorithm.

Due to the risk and cost associated with delivery of logistics to the lunar surface, probabilistic assessments of the exploration systems are a critical analysis component once the exploration trade space has been narrowed ${ }^{2}$. The SAT is performing this analysis using a separate model that can incorporate transportation system risk by probabilistically including failure events and their associated system responses, such as delaying the rest of the scenario. These assessments enable the evaluation of exploration systems on the expected benefit achieved in addition to the planned benefit achieved. The challenges associated with probabilistically loading and reloading a set of flights in response to stochastic events will be discussed at a high level.

\section{Logistics Analysis Overview}

The purpose of CMAT's Logistics Module is to define the logistics requirements for each mission segment and then load those logistics into carrier elements that are delivered prior to the logistics' need date, with the module's concept of operations being outlined in Figure 1. The module takes as input the scenario definition, which consists of the parameters necessary to describe the scenario's set of missions, such as the number of crew delivered, the length of crewed surface duration, the delivery capacity of the transportation system, and the mass of the delivered element systems. The scenario definition is then used to define the mission segments and calculate the logistics requirements for each of them based on the mission parameters, the capabilities of the manifested elements, and a set of assumptions defining crew consumption, Extra-Vehicular Activity (EVA), logistics, science, and In-Situ Resource Utilization (ISRU). The loading algorithm then loads the required logistics onto

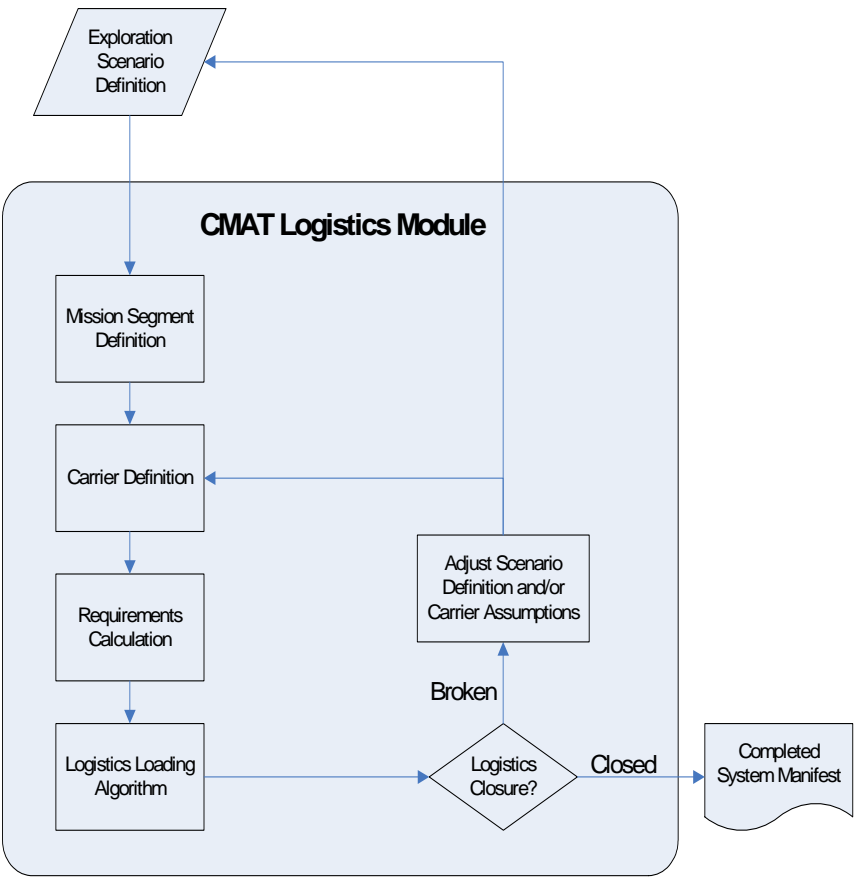

Figure 1: CMAT Logistics Analysis Process each mission within carriers for delivery prior to their date of use. Any cases in which the logistics could not be loaded due to limited capacity are flagged for further attention. Exploration system definition, logistics requirements calculation, and logistics loading are iteratively performed until the exploration system is performing satisfactorily.

The module uses a mission segment based data model to enable the software to handle scenarios with overlapping crewed missions and cargo resupply landings during crewed missions. The module defines segments as the durations between events, with the events modeled being the arrival of a lander or the departure of an ascent stage. Figure 2 shows three examples for how segments are divided out for missions to a single surface location. Case 1 shows a simple set of missions with two crewed missions to the surface and a cargo delivery in between, which results in two crewed segments and two uncrewed segments. Case 2 shows overlapping crewed stays at the single location, which would result in an additional

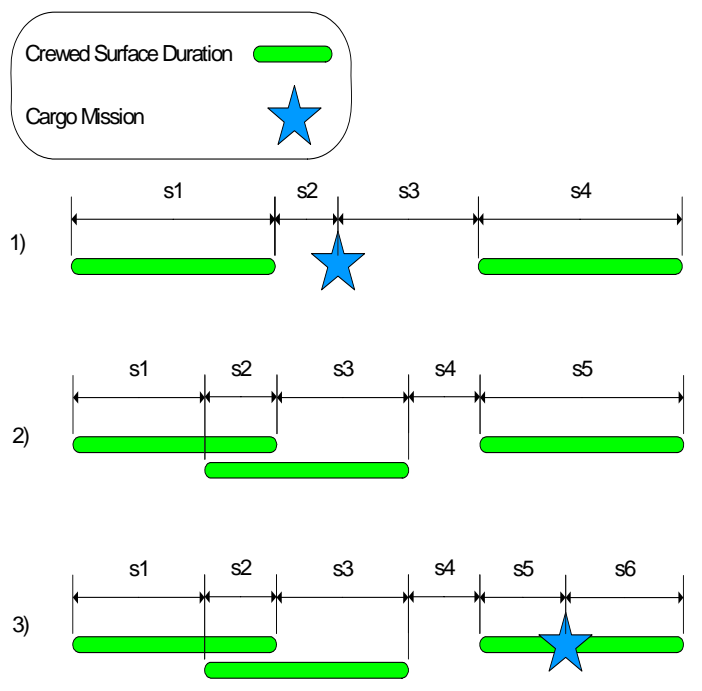

Figure 2: Mission Segment Definition for a Single Surface Location 
segment being created to handle the period of overlap. Similarly, landing a cargo lander during a crewed surface stay will divide the crewed stay into two segments, as illustrated by Case 3 .

The mission segment definition for multiple locations works the same way, except that it is important to note that segments are designated in order of their start date, without regard to location. So in Case 4, as shown in Figure 3, the segments at a given location are not consecutively numbered. In Case 5, a surface transfer has been created between the two locations, further dividing the crewed segments that it intersects with. The transfer itself is also a segment and, as such, the required logistics for the elements involved over the duration of that transfer will also be accounted for by the logistics module.

Once all of the mission segments have been defined, each is assigned to the latest mission arrival prior to the mission segment to create a linkage between the segment's required logistics and the latest mission on which those logistics can be delivered. When probabilistic impacts are accounted for, flight delays can cause delivered logistics to be delivered after their need date. Sufficient contingency supplies can mitigate this issue.

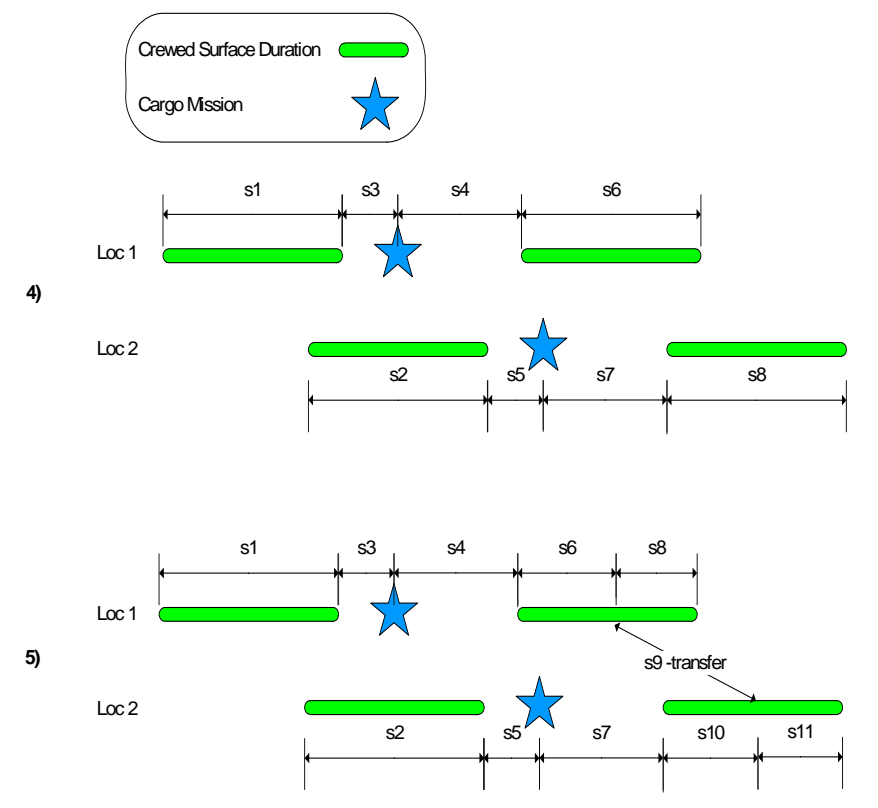

Figure 3: Mission Segment Definition for Multiple Surface Locations

\section{Logistics Requirements}

Any lunar exploration system must account for the logistics required to maintain the elements (rovers, habitats, power systems, etc.) and sustain the crew members on the lunar surface. These logistics requirements are calculated by mission segment and they consist of the logistics necessary to sustain the surface elements and crew, meet defined contingency requirements, and achieve specific objectives, less the logistics produced using ISRU systems. These calculations are performed for each of the eight logistics types defined in CMAT: pressurized crew consumables, pressurized logistics and maintenance (L\&M), unpressurized L\&M, pressurized science, unpressurized science, oxygen, nitrogen, and water.

Contingency requirements can be defined within an exploration scenario to enable the extension of surface stays or to provide an additional logistics cache in the event of an emergency. Required contingencies can be specified for any logistic type on a mission-by-mission basis. This approach enables the contingency requirements to be varied as a function of the planned surface concept of operations and crewed surface durations. Logistics requirements can also be defined for missions to meet specific objectives that are not crew or element related, such as filling a water wall in a habitat to provide radiation protection.

To determine the logistics that need to delivered to the lunar surface, the total logistics required on the surface are offset by the consumables that are produced by ISRU systems or stockpiled on the surface prior to the start of each segment. The ISRU systems currently supported in CMAT include oxygen generation from lunar regolith and water scavenged from the residuals in the lander descent stage propellant tanks. The oxygen generation is treated as an amount generated over time at a specified rate, while the water scavenging is implemented as a single transfer just after each lander has touched down on the surface.

\section{A. Pressurized Crew Logistics}

Pressurized crew logistics account for everything needed for the crew, excluding oxygen, nitrogen, or water. Pressurized crew logistics include food and packaging, cooking and eating supplies, waste collection supplies, hygiene items, clothing, personal items, housecleaning supplies, operations supplies, health care supplies, sleeping provisions, and the pressurized spares required for supporting crew surface EVAs. The consumption rates for these items are listed in Table 1. The rates were provided by Environment Control and Life Support System (ECLSS) 
experts at NASA's Johnson Space Center and Marshall Space Flight Center. The model is updated routinely to reflect the latest information available.

Table 1: Current Assumptions for Pressurized Crew Logistics Consumption Rates

\begin{tabular}{ll} 
Pressurized Crew Item & Consumption Rate \\
\hline Food and Packaging & $2.064 \mathrm{~kg} / \mathrm{crew} /$ day \\
Cooking / Eating Supplies & $0.5 \mathrm{~kg} / \mathrm{crew}$ \\
Waste Collection System Supplies & $0.05 \mathrm{~kg} / \mathrm{crew} /$ day \\
Personal Hygiene Kit & $1.8 \mathrm{~kg} / \mathrm{crew}$ \\
Hygiene Supplies & $0.075 \mathrm{~kg} / \mathrm{crew} /$ day \\
Clothing (with Laundry system) & $13.1 \mathrm{~kg} / \mathrm{crew}$ \\
Clothing (without Laundry system) & $0.46 \mathrm{~kg} / \mathrm{crew} /$ day \\
Personal Stowage & $10 \mathrm{~kg} / \mathrm{crew}$ \\
Disposable Housecleaning Wipes & $0.5 \mathrm{~kg} / \mathrm{crew} /$ day \\
Trash Bags & $0.011 \mathrm{~kg} / \mathrm{crew} /$ day \\
Operations Supplies (diskettes, etc.) & $10 \mathrm{~kg} / \mathrm{crew}$ \\
Health Care Supplies & $0.0856 \mathrm{~kg} / \mathrm{crew} /$ day \\
Medical / Surgical / Dental Supplies & $10 \mathrm{~kg} / \mathrm{mission}$ \\
Sleep Provisions & $9 \mathrm{~kg} / \mathrm{crew}$ \\
EVA Spares & $12 \mathrm{~kg} / \mathrm{mission}$ \\
\hline
\end{tabular}

Currently, the only pressurized crew logistic rate driven by technology selection is the clothing rate, which is based on whether or not a laundry system has been included in the lunar surface habitat. There is potential in the future to handle the housecleaning wipes in a similar way, rather than always assuming disposable wipes are used. The food requirements assume approximately $40 \%$ of the food mass is water. Higher levels of food dehydration are possible, but will require additional water to be supplied.

\section{B. Pressurized \& Unpressurized Logistics and Maintenance}

All surface elements require certain supplies for their maintenance, servicing, repair, or replacement. This L\&M mass includes items that must be replaced on a regular basis (i.e. air filters), replacement masses from the elementto the part-levels, and the tools and supplies necessary to repair elements on the lunar surface. L\&M requirements are broken into two main categories: pressurized supplies for internal L\&M on pressurized elements (i.e. surface habitats, pressurized rovers), and unpressurized supplies for external L\&M on all elements.

The current L\&M requirements are calculated on a per element basis, accounting for the durations each element is in active and dormant operating modes on the surface during a given mission segment. Generally, the current assumptions for these rates are a percentage of the total element mass on a yearly basis, with the elements operating in active mode when crews are present and dormant mode when they are not. Some elements, however, such as power units, unpressurized rovers, or ISRU systems, always operate in their active mode, as they have functions that are crew independent.

The Constellation Program is developing a better understanding of the L\&M masses required to support elements on the surface. Models are being developed that will derive the required L\&M masses with a higher level of fidelity than a percent of element mass per year. These models will include specific replacement/maintenance items, including critical spares, or total element replacement units if necessary. Once the results from these analyses have been made available, they will be used by CMAT to improve the fidelity of the L\&M estimates.

\section{Pressurized \& Unpressurized Science}

Required science masses are currently prescribed on a per-mission basis. CMAT allows pressurized and/or unpressurized science requirements to be specified separately for both crewed and cargo missions. Additional options exists within the tool to specify whether the required science must be delivered on the mission that requires it or whether the logistics loading algorithm can predeploy science for later missions. These options for defining science requirements are included because, at present, the required mass for science delivery for lunar exploration is not well defined. Setting up CMAT to allow requirements to be defined and loaded in multiple ways allows for flexibility in analyzing the impacts of those science requirements as they become better defined.

In some of the exploration scenarios analyzed with CMAT, the mass of the surface elements required early in a given scenario, as well as the logistics required for those early missions, have prevented the delivery of some or all 
of the required science mass on or prior to the start of its assigned mission. To handle such cases, CMAT allows the user to specify the amount of science mass to offload from a given mission's requirements. If there are later missions to the same surface location as the offloaded mission, the science mass that was offloaded is redistributed equally across all later missions to that location. This maintains the same total science delivery to the lunar surface location for the entire exploration scenario, while allowing achievement of early objectives.

\section{Consumables (Oxygen, Nitrogen, \& Water)}

The oxygen, nitrogen, and water required for a given mission are driven by the length of the crewed surface duration, the quantity of EVAs performed on the surface, the level of EVA technology used to operate on the surface, the level of ECLSS technology used in the crewed pressurized environments, and the amount of material produced by ISRU systems.

Oxygen, nitrogen, and water are lost when performing EVAs due to airlock cycling, suit venting, and suit thermal control. Oxygen and nitrogen are lost during the cycling of the airlock, with the quantity lost being calculated from the assumed gas recapture fraction, the number of crew on the surface, the number of crew involved in each EVA, and the number of crew that can use a single airlock at a time. For instance, if there are four crew on the surface and all four are going on an EVA at the same time, but there is only a single two-person airlock available, then that airlock must be cycled a minimum of three times to allow the crew to exit and then reenter the habitat. Additional consumables are required by the crew during their time spent outside of the habitat in EVA suits since the oxygen and water metabolic rates assumed for the crew during EVAs are higher than those for the time spent in a pressurized volume due to higher activity levels.

The ECLSS technology available for use in the crewed pressurized environments can range from a fully open system, where all logistics are brought from Earth and all waste products are vented, to nearly fully closed systems where an initial supply can be recycled continuously with minimal resupply. ECLSS technologies currently represented in CMAT include water recovery, brine recovery, solids drying, oxygen generation from water electrolysis, and $\mathrm{CO}_{2}$ reduction. Three options exist for removing $\mathrm{CO}_{2}$ from the habitat: (1) venting air from the habitat, (2) using a Sabatier reaction and generating methane, and (3) using a Sabatier reaction with a CFR or Bosch process to reclaim the hydrogen from methane, leaving only carbon as the waste product.

\section{1) ECLSS Oxygen}

ECLSS oxygen requirements are driven by crew metabolic rates, venting from the habitat to remove $\mathrm{CO}_{2}$, and leakage from the habitat. The crew metabolic oxygen rate is assumed to be $0.88 \mathrm{~kg} / \mathrm{crew} /$ day for the duration of time they spend in the habitat. Venting is assumed to occur at a rate of $0.16 \mathrm{~kg} / \mathrm{day}$ of air in cases where no other $\mathrm{CO}_{2}$ reduction method has been selected. If $\mathrm{CO}_{2}$ reduction is available, no venting is assumed. Habitat leakage is assumed to occur at $0.05 \%$ of its total volume per day. This leakage rate is a low-fidelity assumption and at some point will need to be updated to reflect the number of hatches or the total pressure seal length present on each pressurized module. Updates to the assumed leakage, however, are not expected to result in significant changes to the consumables requirements.

During the calculation of oxygen and water requirements, some amount of oxygen is generated during the $\mathrm{CO}_{2}$ reduction process, which is added to the oxygen stockpiles and therefore reduces the requirements for a given mission. Additionally, since the packaging for water is lower mass than that for gases, when an oxygen generation (water electrolysis) system is present, all oxygen requirements are converted to their equivalent amount of water and levied as a water requirement.

\section{2) ECLSS Nitrogen}

ECLSS nitrogen requirements are driven by habitat venting and leakage. As previously mentioned for the oxygen requirements, the assumptions for these loss rates are $0.16 \mathrm{~kg} /$ day of air for venting and $0.05 \%$ of total pressurized volume for leakage.

\section{3) ECLSS Water}

The ECLSS water requirements are affected by crew water use rates, the amount of water present in food and waste products, the $\mathrm{CO}_{2}$ reduction method assumed, the amount of water converted to oxygen to meet oxygen requirements, water recovery rates, brine recovery rates, and the amount of water that can be recovered from drying solids. The current assumptions for crew water use rates are displayed in Table 2. The primary water recycling rate 
is calculated based on the total wastewater from all sources, including the water output from any $\mathrm{CO}_{2}$ reduction systems, and the specified ECLSS water recovery rate. The fraction of unrecovered water, referred to as brine, can be partly recovered by an additional brine recovery system. The water in the solid waste, including trash, can also be recovered by a solids drying system. Additionally, to determine the total water requirements, it is necessary to track the $\mathrm{H}_{2} \mathrm{O}, \mathrm{O}_{2} \mathrm{CO}_{2}, \mathrm{CH}_{4}, \mathrm{H}_{2}$, and $\mathrm{C}$ through each portion of the cycle of $\mathrm{CO} 2$ reduction, water electrolysis, and other ECLSS processes.

Table 2: Water Usage Rates

\begin{tabular}{ll} 
Water Usage & Rate \\
\hline Drinking & $2 \mathrm{~kg} / \mathrm{crew} /$ day \\
Food Preparation & $0.5 \mathrm{~kg} / \mathrm{crew} /$ day \\
Hygiene & $0.4 \mathrm{~kg} / \mathrm{crew} /$ day \\
Flushing & $0.5 \mathrm{~kg} / \mathrm{crew} /$ day \\
Laundry & $7.33 \mathrm{~kg} /$ crew/day \\
\hline
\end{tabular}

\section{Logistics Loading Methodology}

Once the logistics required for each mission segment have been determined, those required logistics must be loaded onto the transportation system for delivery to the lunar surface. The logistics loading algorithm takes the requirements linked to each mission, as discussed in the Logistics Analysis Overview, and loads them onto the assigned mission's lander or onto an earlier lander arriving at the same location. This algorithm ensures that the logistics arrive at the appropriate locations prior to their need date.

\section{A. Logistics Carriers}

For transportation to and storage on the lunar surface, logistics must be loaded in carrier containers, which are then loaded onto landers for delivery to the lunar surface. These carriers are elements that have been designed with the capability to carry logistics, with any manually manifested carriers having the capability to carry multiple logistics types. The ability to carry multiple logistics types in a single element allows for a more optimal loading of logistics over the scenario and supports the ability to model how logistics are handled onboard logistics carriers used to supply the ISS, such as the Progress capsule, H-II Transfer Vehicle, and the Automated Transfer Vehicle. For example, a surface habitat could be designed with capacities to deliver both pressurized logistics and water to the lunar surface, rather than manifesting additional water carriers to handle that separately. Each logistics carrier has a capacity that is defined by capability constraints for each type of logistics being carried. Currently, any type of logistics carrier can be manifested individually by the users. However, there are default carriers for unpressurized, oxygen, nitrogen, and water logistics that only carry a single type of logistics and these are the only carriers that are currently manifested automatically by the logistics loading algorithm. These default carriers are described by the properties in Table 3.

Table 3: Unpressurized Carrier Parameters

\begin{tabular}{llll} 
Carrier Type & Mass & Volume & Mass Capacity \\
\hline Unpressurized & $120 \mathrm{~kg}$ & $0.5 \mathrm{~m}^{3}$ & $200 \mathrm{~kg}$ \\
Oxygen & $108 \mathrm{~kg}$ & $0.48 \mathrm{~m}^{3}$ & $108.9 \mathrm{~kg}$ \\
Nitrogen & $108 \mathrm{~kg}$ & $0.48 \mathrm{~m}^{3}$ & $94.8 \mathrm{~kg}$ \\
Water & $34.37 \mathrm{~kg}$ & $0.1 \mathrm{~m}^{3}$ & $74.8 \mathrm{~kg}$ \\
\hline
\end{tabular}

Within pressurized carriers, pressurized logistics are stored in Cargo Transfer Bags (CTBs), currently used on the Space Shuttle for delivering pressurized cargo to the International Space Station, to restrain them during spaceflight. As illustrated in Figure 4, the capacity of CTBs is constrained by limits on the mass and volume of the pressurized logistics to be carried. The capacity of the pressurized carriers is constrained by limits on the number of CTBs that can be loaded in the carrier and the total mass of the loaded CTBs. All of the logistics stored in nonpressurized carriers are subject to mass and volume limits, similar to how the CTBs are loaded. 


\section{B. Loading Algorithm}

The actual loading of logistics onto landers is performed using a loading algorithm that attempts to minimize the unused capacity of each carrier, limiting the total number of carriers delivered to the lunar surface over the course of an entire lunar exploration scenario. The algorithm is able to handle loading multiple types of logistics into different carriers on multiple landers across multiple surface locations, with the structure defining the order the logistics should be loaded, the order the landers should be loaded, and whether the logistics should be delivered on their assigned lander, predeployed to a location on earlier lander, or transferred from another surface location. The current loading algorithm performs the steps outlined in Figure 5, starting with the logistics assigned to the first lunar lander and progressing to those assigned to the last. Within each step, each type of logistic supply is loaded individually, beginning with pressurized logistics and ending with water. The rationale for the steps and the order they are executed are described in the following paragraphs.

The first step is to load as much of the required logistics as possible onto carriers that are manifested on earlier landers going to the same location as the assigned lander. A primary issue with logistics is the large mass of the carriers that are required to sustain the logistics from launch until use. Therefore, to minimize the mass of the carriers, manifested carriers should be filled to their maximum capacity whenever feasible.

In Steps 2-4 the loading algorithm creates carriers to hold logistics on a lander. In Step 2 this creation of new carriers is restricted with rules that are gradually relaxed in Steps 3 and 4 . Step 2 assumes that any already-manifested carrier for a different type of logistics is loaded to its maximum capacity, even if this is not the case. This rule ensures that when a carrier is loaded onto a lander, the lander maintains enough available mass capacity such that any existing carriers on that lander can still be filled to capacity. This approach prevents the algorithm from creating and filling a new carrier such that it prevents logistics from being loaded onto other partially empty carriers on the same lander. Additionally, Step 2 only allows the algorithm to add containers that it can immediately fill to capacity. This rule ensures that a carrier is added first to a lander where it can be filled without preventing existing, partially filled carriers on that same lander from being filled, and also preferentially creates full carriers over partially-filled carriers.

Remaining logistics are loaded in Step 3 by relaxing the rule requiring that added carriers be filled to capacity. Therefore, Step 3 allows the creation of partially-filled carriers that will not prevent other

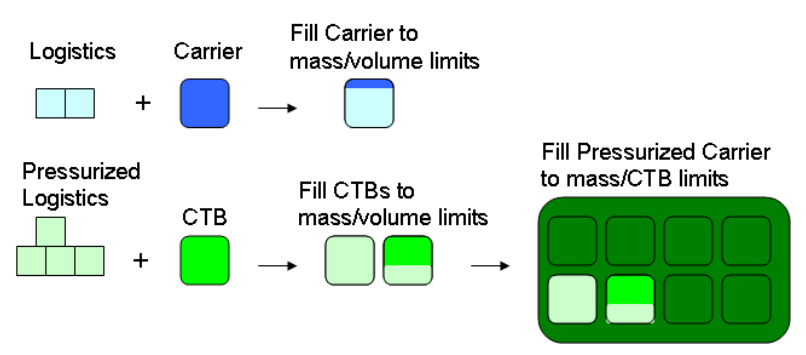

Figure 4: Carrier Loading Methodology
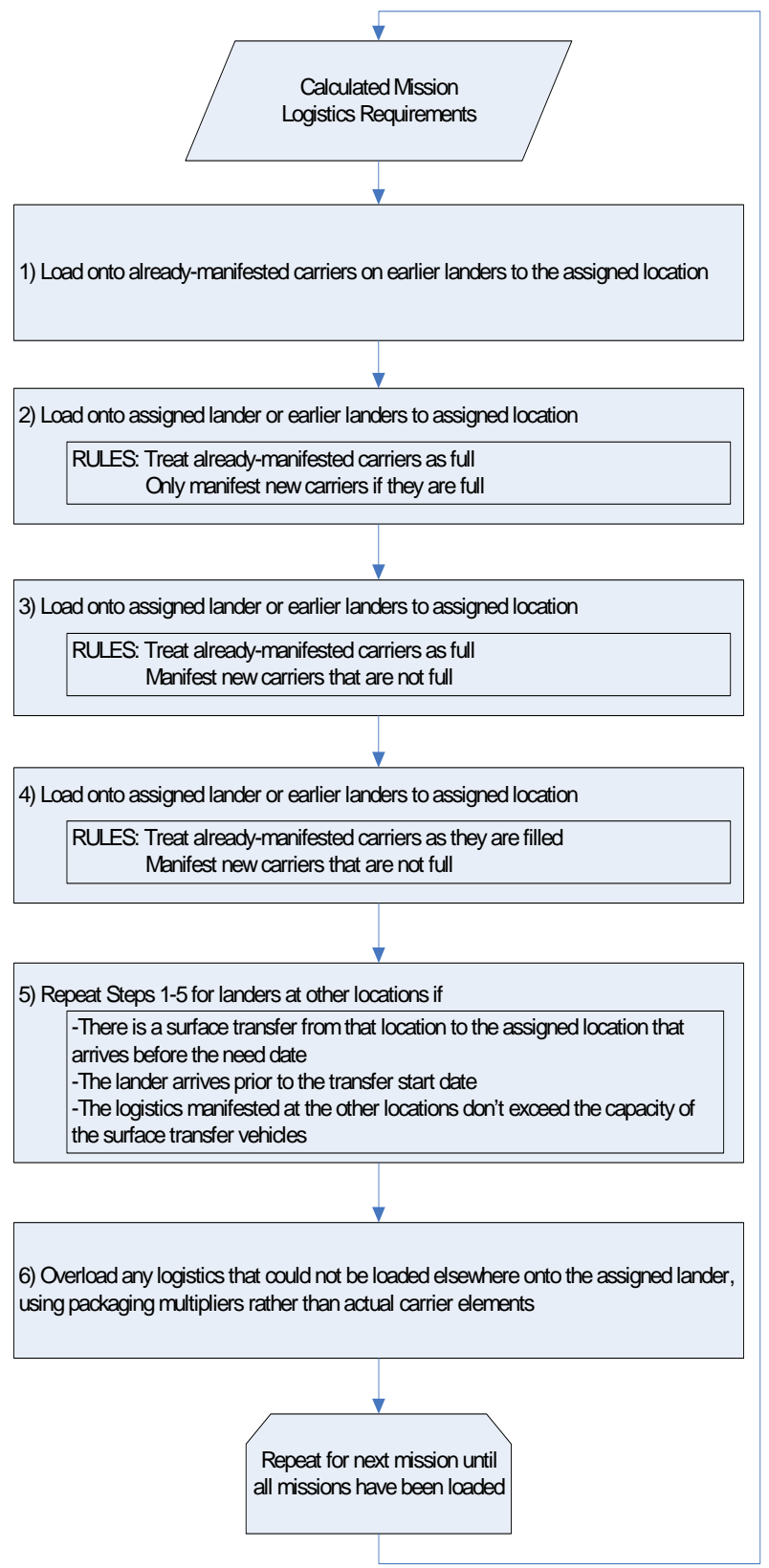

Figure 5: Logistics Loading Algorithm

Each step is performed for all logistics types before moving to the next step. Steps do not execute if all required logistics have been loaded already. 
carriers manifested on that flight from being fully loaded.

Step 4 loads remaining logistics by relaxing the rule that assumes that existing carriers are loaded to maximum capacity. This step allows the creation of partially-filled containers that will prevent carriers on the lander from being filled eventually.

If, after Steps 1-4, there are logistics remaining to be loaded, the algorithm could not find enough capacity in the landers arriving at the assigned location to carry all of the required logistics. In Step 5, the algorithm attempts to load logistics via any surface transfers from another location to the assigned location that arrive before the need date. The logistics can be loaded onto any landers at the other location that arrive prior to the departure of the surface transfer vehicles. The logistics will be loaded following Steps 1-4 again at the surface transfer origin, but will also be subject to the additional restriction that the amount of logistics delivered at the surface transfer origin must not exceed the capacity of the surface vehicle(s) that are performing the surface transfer. If there are still unloaded logistics, Step 5 will also be performed for the transfer origin location to see if the logistics can be delivered through any surface transfers to that location from a third location. The logistics will then be subject to the vehicle limitations used in all surface transfers used to deliver logistics to the assigned location.

Any logistics remaining after Step 5 are logistics that the loading algorithm is unable to load in the given scenario. The loading algorithm handles this situation by forcing those logistics onto the assigned lander, which "overloads" the lander by exceeding its mass capacity. This causes the lander to be displayed as a "broken" lander, which signifies to the user that they need to change the scenario in order to achieve logistics closure for the exploration system being studied.

These modifications to fix a "broken" lander are handled as an iterative process with the user modifying the scenario definition and executing the logistics requirements and loading calculations until closure is achieved. The iterative process can also be used even if logistics closure has already been achieved. This iteration on closed scenarios is generally performed in cases where the analyst needs to maximize use of total delivery capacity, improve achievement of certain performance parameters, or simply improve the loading efficiency of the exploration system. Performing these iterations will generally require the user to adjust the number of crew, modify the surface durations, alter hardware / technology assumptions, or change the element delivery manifest.

This loading algorithm is designed to load quickly and efficiently without using a full optimization routine. Part of the efficiency of the loading process is driven by the order in which logistics are loaded. For example, many exploration systems are driven by the pressurized logistics required to support the crew much more than by the water or gases required. Due to this fact, the current algorithm loads pressurized logistics first and then loads unpressurized logistics, oxygen, nitrogen, and water. The ideal loading prioritization is not the same for all exploration systems, but has been set generally by observing its impact on scenarios that have been analyzed to date.

\section{Limitations of Logistics Loading Algorithm}

This loading methodology does not seek to optimize the logistics loading. Rather, its purpose is to load all required logistics in a manner that is near-optimal, quick, and that will attempt to maximize the unused lander capacity of the exploration system. To some extent this is an issue with calculation time, but is also driven by the need for the analyst to be involved in any optimization or iteration process where adjustments to the scenario may be tied to objectives that are not optimal from a performance perspective. It is likely that this loading methodology could be improved through the use of more sophisticated logic or algorithms.

A related limitation of the logistics module of CMAT, is that often the loading algorithm will deliver logistics a considerable period of time before its need date, neglecting many logistics items' limited lifetimes, crew member specific items, such as clothing, that cannot necessarily be delivered on earlier landers, or L\&M items for a given element that cannot be delivered before the element has been designed. These issues are somewhat mitigated by loading order prioritization that allows the limited lifetime items (pressurized logistics, L\&M, science) prior to loading unlimited lifetime items (oxygen, nitrogen, water). With this loading order, the latter logistics are more likely to be delivered substantially earlier than they are needed. Further, logistics loaded onto earlier landers at their assigned locations are preferentially loaded onto the landers that arrive closest to their need date, which should also serve to minimize the early delivery of logistics.

The logistics module also does not allow multiple carrier sizes to be used for a given logistics type unless they are manually created and manifested. This prevents them from being used very efficiently without extensive user iteration with the logistics module, since currently they cannot be manifested automatically by the loading algorithm. 


\section{Discussion of Probabilistic Logistics Loading}

The described methodology for calculating and loading the required logistics is acceptable for deterministic analysis of an exploration system, but it neglects the substantial risks and potential impacts associated with developing and operating new transportation systems or surface elements. Development has been initiated on a new model that maintains the current rapid trade space investigation capability, while also allowing for in-depth, Monte Carlo-driven probabilistic analysis to be performed on regions of interest within that trade space. Incorporating probabilistics, though, presents additional challenges to a logistics module, particularly as related to the loading algorithm and the speed with which analysis can be performed.

One option is to determine probabilistically when all of the failures will occur for each Monte Carlo case and then load the logistics accordingly. However, this option makes the assumption that failures, and subsequent delays, will be known beforehand and the logistics can be loaded optimally (or near-optimally) with that advance knowledge in hand. A method that is more accurate, but also more costly in terms of computation time, is to load the logistics for the planned scenario, then readjust the loading for the remainder of the scenario after each failure or delay occurs. This is a more realistic approach, but will significantly increase analysis time, particularly when large numbers of Monte Carlo cases are analyzed for each scenario. There may not be a way to avoid this, but a faster and more efficient loading algorithm could reduce the burden on the system associated with calculating and loading logistics.

\section{Conclusion}

The logistics modeling approach as described has been accredited and used to perform deterministic lunar exploration scenario modeling for NASA's Lunar Architecture Team (LAT) and Constellation Architecture Team Lunar (CxAT_Lunar) studies ${ }^{3}$, supporting the assessment of NASA's lunar exploration scenarios from an integrated perspective. This integrated approach has provided decision-makers with an understanding of the performance impacts of: changing priorities, objectives, and assumptions; element-level design choices and technology investments; and differing system (element, architecture, and scenario) approaches. While the current fidelity of the logistics modeling has been sufficient to support the high-level trade space exploration performed in those studies, future analysis requirements will need the ability to perform more in-depth probabilistic assessments of the exploration system to support detailed systems requirements definition.

\section{Acknowledgments}

Thanks to Chel Stromgren, Molly Anderson, Robert Bagdigian, and Jennifer Green for providing the datasets and for their support in developing the requirements algorithms. Thanks to William Cirillo and Kandyce Goodliff for their continued support and driving leadership.

\section{References}

${ }^{1}$ Cirillo, W., Earle, K., Goodliff, K., Reeves, J.D., Andraschko, M., Merrill, R.G., Stromgren, C. Analysis of Logistics in Support of a Human Lunar Outpost. Proceedings of The International Workshop on Modeling and Applied Simulation, September 17-19, 2008, Campora San Giovanni, Amantea, Italy (to be published).

${ }^{2}$ Merrill, R. G., Stromgren, C., Andraschko, M., Cirillo, W., Earle, K., Goodliff, K. A Comparison of Probabilistic and Deterministic Strategic Analysis for Human Space Exploration. Proceedings of AIAA Space 2008 Conference and Exposition. September 9-11, 2008, San Diego, California, USA (to be published).

${ }^{3}$ Cirillo, W., Goodliff, K., Earle, K., Reeves, J.D., Stromgren, C., Andraschko, M., Merrill, R.G. Strategic Analysis Overview. Proceedings of AIAA Space 2008 Conference and Exposition. September 9-11, 2008, San Diego, California, USA (to be published). 